\title{
ORIGINAL
}

\section{EPIDEMIOLOGÍA DE LA TUBERCULOSIS PEDIÁTRICA EN ESPAÑA, 2005-2009}

\section{Elena Rodríguez Valín (1,2), Macarena Garrido Estepa (1), Susana Villarrubia Enseñat (1), Leticia Martín Marcos (1) y Gloria Hernández Pezzi $(1,2)$.}

(1) Área de Vigilancia de Salud Pública. Centro Nacional de Epidemiología. Instituto de Salud Carlos III.

(2) CIBER de Epidemiología y Salud Pública (CIBERESP).

No existe conflicto de interés por parte de los autores

\section{RESUMEN}

Fundamentos: Los últimos datos europeos sobre tuberculosis pediátrica señalan la utilidad de evaluar las tendencias para estudiar la transmisión así como la necesidad de mejorar el diagnóstico microbiológico en estas edades. El objetivo es estudiar la epidemiología de la tuberculosis pediátrica en España, su evolución durante el periodo 2005 2009, y las diferencias respecto con la epidemiología de los adultos.

Métodos: Se utilizaron los datos de la declaración individualizada de tuberculosis a la Red Nacional de Vigilancia Epidemiológica en 2005-2009. Se estudió la tendencia de las tasas en niños y adultos mediante modelos de regresión lineal. Para estudiar los riesgos asociados a la tuberculosis en niños en relación a la de los adultos, se realizó un análisis bivariado y otro multivariado por regresión logística, empleando los datos de 2009

Resultados: En el periodo de estudio se declararon 39.775 casos de tuberculosis, 2.690 de ellos pediátricos $(6,76 \%)$. La tendencia en las tasas de tuberculosis fue ascendente para la edad pediátrica $(y=0,15 x+7,8)$ y descendente para los adultos $(y=-0,28 x+20,2)$. En 2009 las tasas fueron 8,1 casos/100.000 en niños y 18,3/100.000 en adultos. Se encontró en niños una mayor proporción de localizaciones pulmonares $(84 \%$ frente a $76 \%$ ), menor porcentaje de confirmaciones por cultivo $(51 \%$ frente a $82 \%)$ y de casos extranjeros $(25 \%$ frente a $34 \%)$.

Conclusiones: En el periodo de estudio las tasas de tuberculosis pediátrica mostraron una tendencia ligeramente ascendente, mientras que las tasas globales y en adultos la tuvieron descendente. La tuberculosis muestra escenarios diferentes en niños y adultos, lo que hay que tener en cuenta para dirigir las acciones de salud pública.

Palabras clave: Tuberculosis. Niños. Epidemiología. Regresión lineal.

Correspondencia

Elena Rodríguez Valín

Centro Nacional de Epidemiología (pab.12)

Instituto de Salud Carlos III

C/Monforte de Lemos 5

28029 Madrid

ervalin@isciii.es

\section{ABSTRACT}

\section{Epidemiology of Childhood Tuberculosis in Spain: 2005-2009}

Background: European recent data about paediatric tuberculosis point out the importance of evaluate the trends of the disease to study the recent transmission, as well as the necessity of improving the microbiological diagnosis in paediatric cases. The aim of this paper is to study the epidemiology and trend evolution of paediatric tuberculosis in Spain during the period 2005-2009 and to establish the epidemiological differences between adult and paediatric tuberculosis.

Methods: Data reported to the National Surveillance Net (Red Nacional de Vigilancia Epidemiológica) in Spain was checked. Lineal regression was developed to establish the trend of the disease in all, adult and paediatric cases. Bivariate and multivariate logistic regression was used to compare paediatric and adult cases reported in 2009 and estimate the influence of different factors in the development of the disease.

Results: A total 39775,2690 paediatrics $(6.76 \%)$ cases of tuberculosis were reported during 2005-2009 period. Paediatric tuberculosis rates showed a slight increasing tendency $(\mathrm{y}=0.15 \mathrm{x}+7.8)$, while adult rates decrease during the period $(y=-0.28 x+20.2)$. In 2009 , rates were 8.1 and 18.3 cases $/ 100,000$ inhab. for children and adults respectively. Paediatric cases presented higher proportion of pulmonary locations ( $84 \%$ vs. $76 \%$ in adults) and lower percentages of cases confirmed by culture ( $51 \%$ vs. $82 \%$ in adults) and of cases in non-Spanish population (25\% vs. $34 \%)$.

Conclusions: Paediatric tuberculosis rates showed a slight increasing tendency, while global and adult rates decrease slightly during the period. Tuberculosis disease shows different epidemiology in children and adults, what it is important to take into account to design public health interventions.

Key words: Tuberculosis. Children. Epidemiology. Lineal regression. 


\section{INTRODUCCIÓN}

De los 9 millones de casos estimados de tuberculosis que ocurren anualmente en el mundo, 1 millón se producen en menores de 15 años. El control de la tuberculosis pediátrica no ha recibido tanta atención como la de los adultos debido a varias razones, entre ellas la baja proporción de casos bacilíferos en niños, lo que les hace ser potencialmente menos infecciosos ${ }^{1}$. No obstante, hay varios aspectos que resaltan la importancia del control de la tuberculosis en niños. Un caso de tuberculosis en un niño es un evento centinela que indica transmisión reciente en una comunidad. Además, en la edad pediátrica existe una mayor probabilidad de progresión de infección a enfermedad, incluidas las formas graves y extrapulmonares, especialmente en niños menores de 5 años. Adicionalmente, está descrito que los niños son capaces de transmitir la tuberculosis en brotes, tanto en escuelas como en familias ${ }^{2,3}$. Finalmente, los niños infectados representan el reservorio de la enfermedad futura. Por tanto, cualquier descuido en el control de la tuberculosis producirá daños en las generaciones jóvenes, y todos los esfuerzos por reducir el impacto de la tuberculosis a largo plazo deben tener en cuenta el papel que desempeñan los niños enfermos e infecta$\operatorname{dos}^{1,4}$.

En Europa, un reciente trabajo sobre tuberculosis pediátrica (definida como la producida entre los 0-14 años de edad), señala que durante el periodo 2005-2009 las tasas disminuyeron un 2,4\% en la Unión Europea/Área Económica Europea (UE/AEE), si bien existen diferencias entre los países ${ }^{5}$. Para estos países en las últimas recomendaciones europeas se hace énfasis en la necesidad de mejorar el diagnóstico microbiológico y se propone el uso de la razón de tasas niños/adultos como un indicador epidemiológico ${ }^{6}$.

En España, al igual que en el resto de los países de su entorno, las tasas de tuberculosis de los últimos años presentan una tendencia descendente, y los casos en niños representan un porcentaje pequeño respecto al total ${ }^{7,8}$. No obstante, el último informe sobre la situación de la tuberculosis en Europa sugiere que en algunos países, entre los que se encuentra España, se ha producido un aumento en el porcentaje de casos entre los niños nacidos en el país, lo que se atribuye a un aumento en el número de casos en niños clasificados como nacionales, cuyos padres son de origen extranjero, si bien no hay datos suficientes para apoyar esta hipótesis 9 .

Este trabajo tuvo como objetivo estudiar la epidemiología de la tuberculosis pediátrica en España, su evolución en el periodo 2005-2009 y sus diferencias respecto a la tuberculosis en adultos.

\section{MATERIAL Y MÉTODOS}

Para este estudio se utilizaron los datos procedentes de la declaración individualizada de tuberculosis a la Red Nacional de Vigilancia Epidemiológica (RENAVE) correspondientes a los años 2005-2009, disponibles en el Centro Nacional de Epidemiología.

La definición de caso empleada fue la de la RENAVE, que coincide con la europea ${ }^{9}$, y que considera caso de tuberculosis aquel que presenta signos y/o síntomas compatibles con tuberculosis de cualquier localización y prescripción de tratamiento antituberculoso, esté o no confirmado por laboratorio. Se define como tuberculosis pulmonar a la que afecta al parénquima, al árbol traqueobronquial o a la laringe, y extrapulmonar a cualquier otra localización, incluyendo la pleural y la linfática intratorácica cuando no hay afectación pulmonar. En caso de afectación múltiple, la localización pulmonar será considerada siempre como fundamental. Se consideraron casos de tuberculosis pediátrica (TB-PED) los comprendidos entre 0 y 14 años inclusive.

El estudio de las características de los casos se realizó con los datos correspondientes al año 2009, por ser el que presentaba una mejor cumplimentación de las variables. Las varia- 
bles analizadas fueron: edad, sexo, localización de la enfermedad, tratamiento previo, resultados de cultivo y baciloscopia, país de nacimiento y serología VIH. Se calculó la distribución de frecuencias de las distintas variables cualitativas, y las cuantitativas se caracterizaron por su media y desviación estándar. Para estudiar los riesgos asociados a la tuberculosis en niños (TB-PED) en relación a la tuberculosis en adultos (TB-ADUL), se realizó un análisis bivariado y uno multivariado por regresión logística.

Para el cálculo de las tasas de incidencia se emplearon las Estimaciones de la Población Actual del Instituto Nacional de Estadística (INE) para los años 2005-2009. Para estudiar la tendencia de las tasas durante el periodo se utilizaron modelos de regresión lineal y se calcularon los coeficientes de correlación para cada una de las series.

El análisis de los datos se realizó con el paquete estadístico STATA 11.

\section{RESULTADOS}

Entre los años 2005-2009 se declararon a la RENAVE 39.775 casos de tuberculosis, de los que $2.690(6,76 \%)$ fueron en niños. Durante este periodo las tasas de tuberculosis pediátrica en España mostraron una tendencia ligeramente ascendente $\left(\mathrm{R}^{2}=0,13\right)$ con incidencias en torno a 8 casos/100.000 todos los años, excepto en 2008, en el que la tasa fue de 9,3 casos por 100.000 habitantes (figura 1). Comparado con el 2005, sólo en el año 2008 se observó un incremento significativo en el riesgo de tuberculosis pediátrica (ajustado por sexo y lugar de declaración de los casos) $(\operatorname{IRR}=1,18 ; \mathrm{p}=0,006)$.

Por el contrario, la tendencia fue descendente en las tasas de tuberculosis tanto globales $\left(\mathrm{R}^{2}=0,55\right.$; Coef. $=-0,26$; $\mathrm{p}=0,149)$ como en adultos $\left(\mathrm{R}^{2}=0,60\right.$; Coef. $=-0,28 ; p=0,124$ ) (figura 1). En el año 2009 las tasas de incidencia de tuberculo- sis fueron de 8,1 casos/100.000 habitantes en niños y 18,3 casos/100.000 habitantes en adultos (tasa global para todas las edades 16,8). El IRR ajustado mostró una disminución significativa en el riesgo de contraer la enfermedad en 2009 en comparación con los datos del 2005, tanto para los datos globales (IRR $=0,94$; $\mathrm{p}<0,001)$ como para el grupo de adultos (IRR $=0,93 ; \mathrm{p}<0,001)$, corroborando el dato obtenido mediante regresión lineal.

Características de los casos de tuberculosis pediátrica

En el año 2009 se declararon a la RENAVE 7.719 casos de tuberculosis. Se utilizaron para nuestro estudio 7.717 casos de los que se conocía la edad. De éstos, 549 (7\%) fueron TB-PED y 7.168 (93\%) fueron TB-ADUL.

Del total de casos pediátricos, 287 (52 $\%$ ) fueron en menores de 5 años y 262 (48\%) tenían entre 5 y 14 años. Las características de los casos de tuberculosis en niños en relación con los adultos se muestran en las tablas 1 y 2 .

Respecto al sexo, los casos de TB-PED se reparten uniformemente entre ambos, aunque la probabilidad de ser del sexo femenino es superior a la de los casos de TB-ADUL $(\mathrm{ORa}=1,9, \mathrm{IC} 95 \%=1,3-2,6)$ (tabla 2).

Los casos pediátricos presentan un porcentaje más elevado de localizaciones pulmonares que los adultos $(84 \%$ frente a $76 \%, \mathrm{ORa}=2,1, \mathrm{IC} 95 \%=1,4-3,2)$ (tabla $2)$. Analizando las localizaciones fundamentales con más detalle (tabla 1), se observa que los casos de TB-PED presentan además porcentajes superiores de localizaciones respiratorias, linfáticas y meníngeas.

Respecto al tratamiento previo, menos del 1\% (3) de los casos TB-PED habían 
Figura 1

Tasas de incidencia de tuberculosis totales y por grupos de edad. España, 20052009

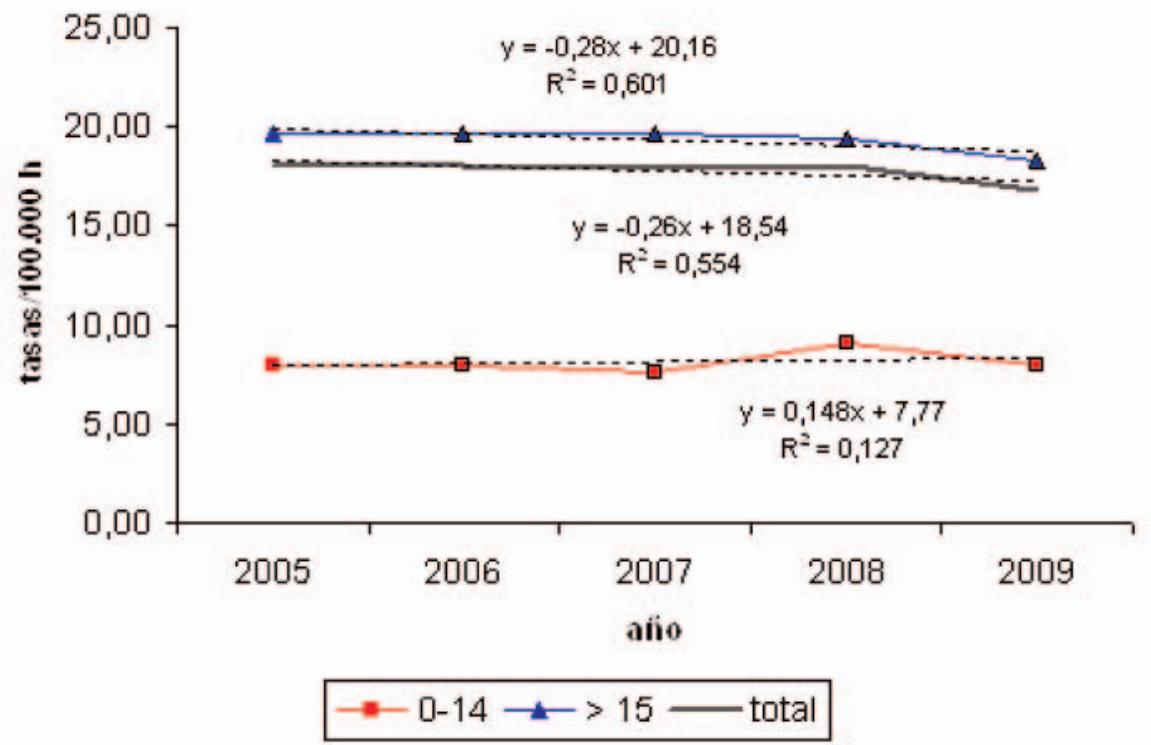

Tabla 1

Localizaciones de la tuberculosis en los casos pediátricos y no pediátricos España, 2009

\begin{tabular}{|l|c|c|c|c|c|c|}
\hline & $\mathbf{0 - 1 4}$ años & & $\mathbf{7 1 5}$ años & & total & \\
\hline localización & $\mathrm{n}$ & $\%$ & $\mathrm{n}$ & $\%$ & $\mathrm{n}$ & $\%$ \\
\hline Pulmonar & 433 & $\mathbf{7 8 , 8 7}$ & 5181 & 72,28 & 5614 & 72,75 \\
\hline Linfática & 27 & $\mathbf{4 , 9 2}$ & 246 & 3,43 & 273 & 3,54 \\
\hline Respiratoria s.p. & 24 & $\mathbf{4 , 3 7}$ & 169 & 2,36 & 193 & 2,50 \\
\hline Meningea & 14 & $\mathbf{2 , 5 5}$ & 90 & 1,26 & 104 & 1,35 \\
\hline Pleural & 2 & 0,36 & 234 & 3,26 & 236 & 3,06 \\
\hline Digestiva & 2 & 0,36 & 19 & 0,27 & 21 & 0,27 \\
\hline Diseminada & 2 & 0,36 & 81 & 1,13 & 83 & 1,08 \\
\hline Osteoarticular & 1 & 0,18 & 55 & 0,77 & 56 & 0,73 \\
\hline Genitourinaria & 0 & 0,00 & 69 & 0,96 & 69 & 0,89 \\
\hline Extrarrespiratoria. s.p. & 44 & 8,01 & 1024 & 14,29 & 1068 & 13,84 \\
\hline Total & 549 & 100,00 & 7168 & 100,00 & 7717 & 100,00 \\
\hline
\end{tabular}

s.p: sin especificar. 
Tabla 2

Comparación de los casos de tuberculosis pediátrica y no pediátrica. Resultados del análisis univariado y multivariado. España, 2009

\begin{tabular}{|c|c|c|c|c|c|c|c|c|c|}
\hline & & $0-14$ años & $>15$ años & $\begin{array}{c}\text { Análisis } \\
\text { univariado }\end{array}$ & & & $\begin{array}{c}\text { Análisis } \\
\text { multivariado }\end{array}$ & & \\
\hline Variable & & $\mathrm{n}(\%)$ & $\mathrm{n}(\%)$ & $\mathrm{ORc}$ & IC $95 \%$ & $p$ & $\mathrm{ORa}$ & IC $95 \%$ & $p$ \\
\hline \multirow[t]{2}{*}{ Sexo } & hombre & $277(50,6)$ & $4491(63,3)$ & - & & & - & & \\
\hline & mujer & $270(49,4)$ & $2608(36,7)$ & 1,70 & $(1,40-2,01)$ & $<0,001$ & 1,87 & $(1,33-2,65)$ & $<0,001$ \\
\hline \multirow[t]{2}{*}{ Localización } & extrapulmonar & $90(16,4)$ & $1700(23,7)$ & - & & & - & & \\
\hline & pulmonar & $459(83,6)$ & $5468(76,3)$ & 1,58 & $(1,25-2,02)$ & $<0,001$ & 2,11 & $(1,36-3,25)$ & 0,001 \\
\hline \multirow{2}{*}{ Result, cultivo } & negativo & $133(48,9)$ & $945(18,4)$ & - & & & - & & \\
\hline & positivo & $139(51,1)$ & $4193(81,6)$ & 0,24 & $(0,18-0,30)$ & $<0,001$ & 0,33 & $(0,23-0,48)$ & $<0,001$ \\
\hline \multirow[t]{2}{*}{ Result, baciloscopia } & negativo & $255(89,2)$ & $2771(50,9)$ & & & & & & \\
\hline & positivo & $31(10,08)$ & $2671(49,5)$ & 0,13 & $(0,08-0,18)$ & $<0,001$ & 0,10 & $(0,05-0,20)$ & $<0,001$ \\
\hline \multirow[t]{2}{*}{ Pais nacimiento. } & España & $406(74,9)$ & $4692(66,4)$ & - & & & - & & \\
\hline & otro & $136(25,1)$ & $2374(33,6)$ & 0,66 & $(0,54-0,81)$ & $<0,001$ & 0,55 & $(0,37-0,81)$ & 0,002 \\
\hline \multirow[t]{2}{*}{ Result. VIH } & negativo & $264(98,5)$ & $3318(87,9)$ & - & & & - & & \\
\hline & positivo & $4(1,5)$ & $456(12,1)$ & 0,11 & $(0,03-0,29)$ & $<0,001$ & 0,17 & $(0,05-0,53)$ & 0,002 \\
\hline \multirow[t]{2}{*}{ Tto.previo } & no & $528(99,4)$ & $6290(93,9)$ & - & & & - & & \\
\hline & si & $3(0,6)$ & $411(6,1)$ & 0,09 & $(0,02-0,26)$ & $<0,001$ & - & - & \\
\hline
\end{tabular}

Tto $=$ tratamiento antituberculoso. ORc $=$ Odds Ratio cruda. Ora $=$ Odds Ratio ajustada. IC95\%= Intervalo de confianza al $95 \%$.

sido tratados previamente, frente al $6 \%$ de los casos de TB-ADUL (tabla 2). Los tres niños que habían recibido tratamiento previo antituberculoso tenían edades entre 10-13 años, y todos presentaban localización pulmonar.

En el año 2009 se declararon 4 (1,5\%) casos VIH positivos entre los TB-PED, siendo este porcentaje muy superior en los adultos (12\%) (ORa $=0,2$; IC $95 \%=$ $0,05-0,53$ ) (tabla 2). Estos 4 niños con coinfección TB-VIH tenían diferentes edades distribuidas entre 0-9 años, y sólo 1 presentaba localización pulmonar. Hay que señalar que el porcentaje de casos sin información acerca de la prueba del VIH es cercano al $50 \%$, tanto en los casos de TB-PED como en los de TB-ADUL.

Otra característica de los casos de tuberculosis en niños es la escasa información microbiológica en relación con los casos en adultos, y el menor porcenta- je de casos confirmados. Así, el porcentaje de casos con información de resultados de laboratorio en 2009 es de 46\% (249) en los TB-PED frente a $69 \%(4.926)$ en los TB-ADUL. De los que tienen información, el porcentaje de casos confirmados por cultivo es muy inferior en los casos pediátricos $(51 \%$ frente al $82 \%$ de los TBADUL, ORa $=0,3, \mathrm{IC} 95 \%=0,2-0,5)$. Respecto a la baciloscopia, de los que se tiene información, el 11\% de los TB-PED tienen baciloscopia positiva, frente al $49 \%$ d e 1 o s T B - A D U L ( O R a = 0, 1, IC95\% $=0,05-0,20)$ (tabla 2$)$.

Los casos de tuberculosis infantil presentan una menor proporción de extranjeros que los casos en adultos $(25 \%$ en TBPED, frente al $34 \%$ en TB-ADUL, $\mathrm{ORa}=0,5, \mathrm{IC} 95 \%=0,4-0,8)($ tabla 2$)$. Entre los años 2005-2009 el número de casos nacidos fuera de España ha sufrido un aumento significativo, tanto en niños como en adultos (en niños $\mathrm{R}^{2}=0,9$, 
Figura 2

Casos declarados de tuberculosis por grupo de edad y país de nacimiento. España, 2005-2009

\section{0-14 años}
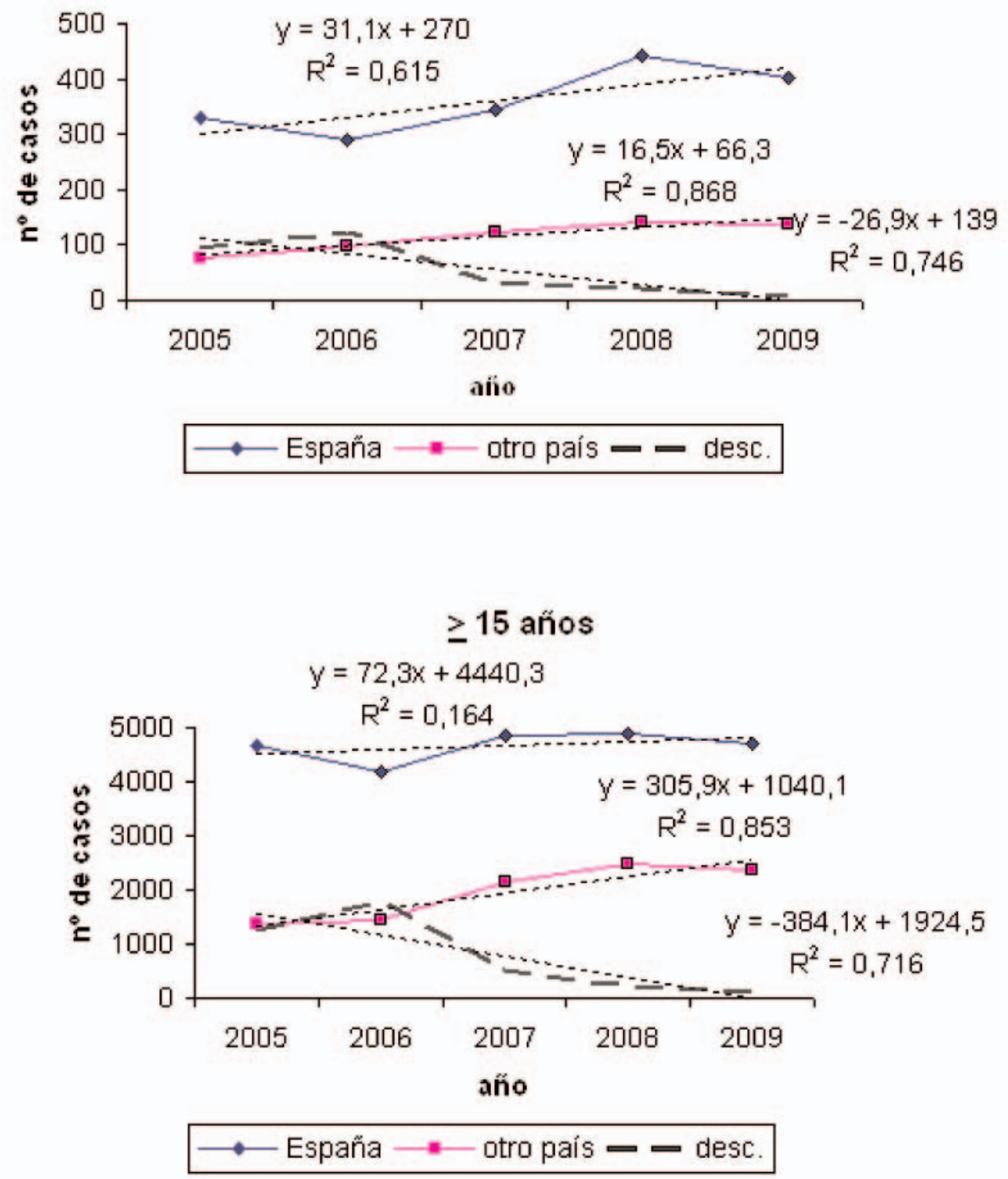
$\mathrm{p}=0,021$ y en adultos $\left.\mathrm{R}^{2}=0,8, \mathrm{p}=0,025\right)$ (figura 2). El número de casos nacidos en España también ha aumentado en niños en el mismo periodo, y en adultos ha permanecido estable, aunque de forma no significativa en ambos $\left(R^{2}=0,6\right.$, $\mathrm{p}=0,12$ y $\mathrm{R}^{2}=0,2, \mathrm{p}=0,49$ en niños $\mathrm{y}$ adultos respectivamente).

La distribución de los casos de TBPED por edad y país de nacimiento declarados en 2009 muestra que, en menores de 5 años, entre el 80 y el $90 \%$ de los casos son nacidos en España, mientras que a partir de los 5-6 años va aumentando progresivamente la proporción de niños nacidos en otros países (tabla 3 ).
La información que especifica el país de nacimiento, la cual no es obligatoria, se obtuvo a partir de los datos procedentes de 5 Comunidades Autónomas (CCAA) que representan el $37 \%$ de los casos de toda España. Esta información está disponible en 1.397 de $\operatorname{los} 2.511$ casos extranjeros declarados en el año 2009 (56\%). De estos 1.397 casos nacidos fuera de España, 70 son en niños, y los países de procedencia más frecuentes fueron Marrue$\cos (34 \%)$, Rumania (16\%), Bolivia $(10 \%)$ y Ecuador $(9 \%)$ de los TB-PED. Los principales países de procedencia de los 1.327 casos adultos fueron los mismos (Marruecos, Rumania, Bolivia y Ecuador representaron el 17\%, 11\%,

Tabla 3

Distribución de los casos de tuberculosis pediátrica por edad y país de nacimiento España, 2009

\begin{tabular}{|c|c|c|c|c|c|}
\hline \multicolumn{6}{|c|}{ País de nacimiento } \\
\hline & total & España & & Otro país & \\
\hline años & $\mathrm{n}$ & $\mathrm{n}$ & $\%$ & $\mathrm{n}$ & $\%$ \\
\hline 0 & 43 & 35 & 81,40 & 8 & 18,60 \\
\hline 1 & 66 & 62 & 93,94 & 4 & 6,06 \\
\hline 2 & 61 & 53 & 86,89 & 8 & 13,11 \\
\hline 3 & 60 & 50 & 83,33 & 10 & 16,67 \\
\hline 4 & 52 & 44 & 84,62 & 8 & 15,38 \\
\hline 5 & 33 & 26 & 78,79 & 7 & 21,21 \\
\hline 6 & 36 & 23 & 63,89 & 13 & 36,11 \\
\hline 7 & 27 & 17 & 62,96 & 10 & 37,04 \\
\hline 8 & 26 & 21 & 80,77 & 5 & 19,23 \\
\hline 9 & 29 & 19 & 65,52 & 10 & 34,48 \\
\hline 10 & 22 & 10 & 45,45 & 12 & 54,55 \\
\hline 11 & 21 & 10 & 47,62 & 11 & 52,38 \\
\hline 12 & 15 & 10 & 66,67 & 5 & 33,33 \\
\hline 13 & 27 & 14 & 51,85 & 13 & 48,15 \\
\hline 14 & 24 & 12 & 50,00 & 12 & 50,00 \\
\hline total & 542 & 406 & 74,91 & 136 & 25,09 \\
\hline
\end{tabular}


$13 \%$ y $8 \%$ de los casos TB-ADUL respectivamente), si bien se encuentran repartidos entre una mayor diversidad de países.

\section{DISCUSIÓN}

Las tasas de tuberculosis en niños han experimentado un ligero ascenso en España durante el periodo 2005-2009, con un aumento de riesgo significativo en el año 2008 en relación a 2005. Este aumento, ya descrito en algunas $\mathrm{CCAA}^{10-12}$ puede deberse en parte a la presencia de brotes, si bien los datos utilizados en nuestro estudio, procedentes de la declaración individualizada, no nos permiten discriminar cuáles de esos casos están asociados a un brote. En el año 2009 la incidencia volvió a bajar, si bien se mantuvo ligeramente superior a la de años previos a 2008. Este es un fenómeno característico de los países de baja incidencia de tuberculosis, en los que las tasas pediátricas se ven muy afectadas por los brotes o por circunstancias puntuales, y no por la tendencia general de la población como pasa en los países de elevada incidencia ${ }^{4,5}$. España se comportaría en este caso como un país de baja incidencia, en el que la tendencia es descendente en las tasas de tuberculosis en adultos y en la población general, mientras que en los niños es ascendente, lo que indica escenarios epidemiológicos diferentes.

Para estudiar las características de la tuberculosis en niños y sus diferencias con la de los adultos, se han empleado los datos del año 2009 por ser el que presentaba información más completa. En ese año, los casos de tuberculosis en niños representaron el $7 \%$ del total de casos declarados. Este porcentaje es superior a la media europea y similar al de países como Bulgaria, Grecia o Bélgica ${ }^{5,9}$.

Respecto al sexo, los casos de tuberculosis infantil presentan un equilibrio entre ambos sexos en cuanto a número, lo que les diferencia de la tuberculosis en adultos, que afecta predominantemente a los hombres. Esto es similar a los resultados de otros estudios y es coherente con la epidemiología de la enferme- dad, puesto que los factores de riesgo que afectan a los hombres todavía no están presentes en la edad pediátrica ${ }^{13-15}$.

La localización fundamental de la enfermedad en niños, al igual que en los adultos, es la pulmonar, si bien dentro de las localizaciones extrapulmonares los niños tienen mayores porcentajes de tuberculosis meníngeas y linfáticas que los adultos, lo que está descrito en la literatura ${ }^{13,15-17}$.

En nuestro estudio, el número de casos de TB-PED que habían recibido un tratamiento previo antituberculoso fue muy escaso. No obstante, el tratamiento previo es un factor de riesgo para el desarrollo de resistencia primaria en tuberculosis y, aunque en este estudio no disponemos de información sobre resistencias, diversos autores en España y otros países señalan tasas elevadas de resistencia primaria en niños inmigrantes ${ }^{13,15,18,19}$, de ahí la importancia de la recogida de esta información y de la conveniencia de realizar antibiograma en todos los casos, tal como señalan las recomendaciones nacionales ${ }^{20-22}$.

Respecto al VIH, España ocupa el séptimo lugar entre los nueve países europeos que notifican prevalencias de coinfección TB-VIH superiores al $5 \%{ }^{9}$. Aunque en nuestro estudio el porcentaje de niños coinfectados es muy pequeño y no parece representar un problema en nuestro país, no se puede menospreciar, ya que ser seropositivo aumenta el riesgo de enfermedad diseminada y, por tanto, de mayor gravedad. También hay evidencias de que los niños infectados por el VIH tienen mayor probabilidad de estar expuestos a adultos bacilífe$\operatorname{ros}^{1}$.

Los resultados de nuestro estudio indican que entre los casos de TB-PED que disponen de información de laboratorio, la mitad están confirmados por cultivo y el $10 \%$ tienen baciloscopia positiva, frente al $80 \%$ y $50 \%$ de los TB-ADUL respectivamente. El porcentaje de confirmaciones por cultivo es superior al de la media europea, pero similar al de otros paí- 
$\operatorname{ses}^{5,18}$, y este dato junto con el bajo porcentaje de baciloscopias positivas pone en evidencia la dificultad del diagnóstico de la tuberculosis infantil y la necesidad de mejorarlo ${ }^{16,22}$. Diversos trabajos han señalado una subdeclaración de los casos de tuberculosis pediátrica, así como dificultades en su manejo clínico ${ }^{19,23,24}$. En España no existen estudios representativos en este sentido, si bien siendo conscientes de los retos y las dificultades que presenta el diagnóstico de la tuberculosis en los niños, recientemente se han actualizado las recomendaciones nacionales ${ }^{22}$. También hay que tener en cuenta que los datos de vigilancia, en los que se basa este trabajo, solo recogen como métodos de diagnóstico la baciloscopia y el cultivo, por lo que no se ven reflejados otros métodos que pueden ser empleados para el diagnóstico de la tuberculosis infantil ${ }^{25}$.

En el periodo de nuestro estudio en España se ha producido un aumento en el número de casos de tuberculosis de origen extranjero, si bien este aumento ha ido ligado a una mejora de la declaración. La variable "país del caso", incluida en la declaración individualizada y desglosada en tres categorías (España, fuera de España y desconocido), fue de escasa cumplimentación hasta el año 2007, en el que se produjo una mejora de la declaración. A partir de entonces, el número de casos nacidos fuera de España ha ido en aumento. En el año 2009 el porcentaje de casos de tuberculosis nacidos fuera de España fue de $34 \%$ en adultos y de $25 \%$ en niños. Diversos estudios han señalado que muchos casos de tuberculosis en niños clasificados como nacionales se producen en núcleos de inmigrantes, los cuales han adquirido la infección por contacto con un adulto conviviente o por viajes a países de alta endemia tuberculosa ${ }^{1,5,17}$. En otros estudios de ámbito local realizados en España, se señala el incremento notable del porcentaje de niños inmigrantes con tuberculosis, y se destaca el hecho de que cada vez es mayor el porcentaje de ellos en los que el adulto índice es un familiar no conviviente, $\mathrm{u}$ otras personas adultas como cuidadoras ${ }^{15}$. Los datos nacionales utilizados para nuestro estudio no permiten llegar a ese nivel de detalle al no disponer de información sobre país de origen de los padres, si bien se ha visto que el porcentaje de casos de tuberculosis nacidos fuera de España ha aumentado tanto en niños como en adultos. También observamos que el porcentaje de niños extranjeros aumenta progresivamente a partir de los 5-6 años, edad a partir de la cual la escolarización es obligatoria, lo que nos hace pensar en el riesgo añadido de la escolarización. Respecto a los países de procedencia de los casos nacidos fuera de España, la información procede de cinco CCAA que reciben inmigración fundamentalmente del Norte de África, América del Sur y Europa del Este, por lo que los resultados señalan a Marruecos, Rumania y algunos países de América Latina como los que representan la mayor carga de casos extranjeros, tanto en niños como en adultos. Estos datos no son representativos de la población general y se pueden considerar como una aproximación al perfil de la inmigración en nuestro país ${ }^{26-28}$.

Concluyendo, las tasas de tuberculosis en el periodo 2005-2009 en España muestran una tendencia ligeramente ascendente en la edad pediátrica, y descendente en las tasas globales y de adultos. A lo largo de ese periodo se ha observado un aumento significativo del número de casos nacidos en otros países, ligado probablemente a una mejora en la declaración.

Los casos de TB-PED en España se encuentran distribuidos uniformemente entre ambos sexos y presentan un mayor porcentaje de localizaciones pulmonares que los casos en adultos, y un menor porcentaje de confirmaciones de laboratorio. Estas características son similares a los países europeos de nuestro entorno y ponen de manifiesto la necesidad de mejorar el diagnóstico de la tuberculosis en los niños, ya señalada en otros trabajos.

Los casos de TB-PED menores de 5 años son en su mayoría españoles, mientras que a partir de esa edad va aumentando el porcentaje de niños extranjeros, lo que pone de relieve la importancia del estudio de contactos y el 
control de la tuberculosis en centros escolares y en población inmigrante. Una recomendación futura para poder caracterizar mejor el riesgo de los casos de TB-PED a partir de los datos de vigilancia, sería el disponer de información en la declaración individualizada sobre si el caso está asociado a un brote y sobre el lugar de origen de los padres. Finalmente, la inclusión de indicadores epidemiológicos relacionados con la tuberculosis infantil en los programas nacionales, junto con las medidas anteriores, permitirían avanzar en el control de la tuberculosis en los niños.

\section{AGRADECIMIENTOS}

A los Servicios de Epidemiología de las CCAA, que suministran sus datos de forma regular a la Red Nacional de Vigilancia Epidemiológica, y que permiten disponer de una información sólida y representativa a nivel estatal. Y en general a los profesionales de Salud Pública (profesionales de atención primaria y especializada) que contribuyen con su trabajo a mejorar la calidad del sistema de vigilancia y control de la tuberculosis en España

\section{BIBLIOGRAFÍA}

1. Nelson LJ, Wells CD. Global epidemiology of childhood tuberculosis. Int J Tuberc Lung Dis. 2004; $8(5): 636-647$.

2. Alvarez-Castillo MC, Cano Escudero S., Taveira Jimenez JA. Microepidemias de tuberculosis en centros escolares. ¿Cómo seleccionar los contactos? Gac Sanit. 2007; 21(6):465-470.

3. Nelson JL, Moreno A, Orcau A, Altet N, MartinezRoig A, Cayla JA et al. Transmission of childhood tuberculosis risk factors associated with an unidentified index case and outbreak evolution in Barcelona (1987-2007). Pediatr Infect Dis J. 2010; 29(9):876-879.

4. Haas W. High time to tackle childhood tuberculosis. Euro Surveill. 2011; 16(12).

5. Sandgren A, Hollo V, Quinten C, Manissero D. Childhood tuberculosis in the European Union/European Economic Area, 2000 to 2009. Euro Surveill. 2011; 16(12).
6. European Centre for Disease Prevention and Control S. Progressing towards TB elimination. A follow-up to the Framework Action Plan to fight tuberculosis in the European Union. Stockholm: European Centre for Disease Prevention and Control; 2010.

7. Hollo V, Zucs P, Kodmon C, Sandgren A, Manissero D. Marking 15 years of efforts towards a comprehensive European TB surveillance system: the epidemiological situation of TB in the EU/EEA in 2009. Euro Surveill. 2011; 16(12).

8. Rodríguez E, Villarrubia S, Díaz O, Hernández G, Tello O. Situación de la tuberculosis en España. Casos de tuberculosis declarados a la Red Nacional de Vigilancia Epidemiológica en 2009. Bol Epidemiol Semanal. 2010; 18(22):213-216.

9. European Centre for Disease Prevention and Control/WHO Regional Office for Europe: Tuberculosis surveillance in Europe 2009. Stockholm: European Centre for Disease Prevention and Control; 2011.

10. Dirección General de Salud Pública. Informe de tuberculosis. Comunitat Valenciana. Año 2008. Valencia: Consellería de Sanitat; 2009.

11. Generalitat de Catalunya.Departament de Salut. Informe anual 2008. Situación epidemiológica y tendencia de la endemia tuberculosa en Cataluña. Barcelona: Consellería de Sanitat; 2009.

12. Xunta de Galicia.Consellería de Sanidad. Informe de la tuberculosis en Galicia: Características de los casos de tuberculosis de Galicia en el año 2009. Evolución en el periodo 1996-2009. Santiago de Compostela: Xunta de Galicia;2011.

13. Abubakar I, Laundy MT, French CE, Shingadia D. Epidemiology and treatment outcome of childhood tuberculosis in England and Wales: 19992006. Arch Dis Child. 2008; 93(12):1017-1021.

14. Allotey P, Gyapong M. Gender in tuberculosis research. Int J Tuberc Lung Dis. 2008; 12(7):831836.

15. del Rosal T, Baquero-Artigao F, Garcia-Miguel MJ, Mendez-Echevarria A, Lopez G, Aracil FJ et al. Impact of immigration on pulmonary tuberculosis in Spanish children: a three-decade review. Pediatr Infect Dis J. 2010; 29(7):648-651.

16. Rigouts L. Diagnosis of childhood tuberculosis. Eur J Pediatr. 2009; 168:1285-1290. 
17. Ruwende JE, Sanchez-Padilla E, Maguire H, Carless J, Mandal S, Shingadia D. Recent trends in tuberculosis in children in London. J Public Health. (Oxf) 2011; 33(2):175-181.

18. Menzies HJ, Winston CA, Holtz TH, Cain KP, Mac Kenzie WR. Epidemiology of tuberculosis among USand foreign-born children and adolescents in the United States, 1994-2007. Am J Public Health. 2010; 100(9):1724-1729.

19. Teo SS, Riordan A, Alfaham M, Clark J, Evans MR, Sharland M et al. Tuberculosis in the United Kingdom and Republic of Ireland. Arch Dis Child. 2009; 94(4):263-267.

20. Gonzalez-Martin J, Garcia-Garcia JM, Anibarro L, Vidal R, Esteban J, Blanquer R et al. Documento de consenso sobre diagnóstico, tratamiento y prevención de la tuberculosis. Sociedad Española de Enfermedades Infecciosas y Microbiología Clínica y Sociedad Española de Neumología y Cirugía Torácica. Arch Bronconeumol. 2010; 46(5):255-274.

21. Ministerio de Sanidad y Política Social. Plan para la prevención y control de la tuberculosis en España. Propuesta del Grupo de trabajo de expertos en tuberculosis y del Grupo de trabajo de Salud Pública para la prevención y control de la tuberculosis, aprobada por la Comisión de Salud Pública del Consejo Interterritorial del Sistema Nacional de Salud de España (15 de noviembre de 2007). Madrid: Ministerio de Sanidad y Política Social; 2008.

22. Moreno-Perez D, Andres MA, Altet GN, BaqueroArtigao F, Escribano MA, Gomez-Pastrana DD et al. Diagnóstico de la tuberculosis en la edad pediátrica. Documento de consenso de la Sociedad Española de Infectología Pediátrica y de la Sociedad Española de Neumología Pediátrica. An Pediatr. (Barc) 2010; 73(3): 143 .

23. Siegel D, Song X, Klontz K, Pastor W, Singh N. Epidemiology of childhood tuberculosis: use and evaluation of the pediatric health information system to assess local and national incidence. Pediatr Infect Dis J. 2011; 30(5):428-430.

24. Teo SS, Alfaham M, Evans MR, Watson JM, Riordan A, Sonnenberg P et al. An evaluation of the completeness of reporting of childhood tuberculosis. Eur Respir J. 2009; 34(1):176-179.

25. Pérez E, González F, Monparler P, Huertas I, Carbó $\mathrm{R}$, de la Encarnación A. Uso de la TC en el diagnóstico de tuberculosis infantil en la Comunidad Valenciana. Años 2004-2010. Gac Sanit. 2011; 25(Espec Congr 2): 148 .
26. Registro Regional de casos de tuberculosis de la Comunidad de Madrid, informe del año 2009. Bol Epidemiol Comunidad Madrid. 2010; 16(10): 3-36.

27. Generalitat de Catalunya. Departament de Salut. Informe anual 2009. Situación epidemiológica y tendencia de la endemia tuberculosa en Cataluña. Departament de Salut; 2010.

28. Generalitat Valenciana. Informe de tuberculosis. Comunitat Valenciana. Año 2009. Valencia: Conselleria de Sanitat;2010. 